\title{
Transverse Vibrations of an Elliptic Plate.
}

\author{
By Mr. Jyotirmara Ghosh, M.A. Dacca University.
}

(Received 27th Junuary 1926. Read 5th February 1926.)

\section{$\S 1$. Introductory.}

Since the publication of the memoir of Mathieu on the transverse vibrations of an elliptic membrane, the subject has been discussed by many authors from different points of view. But the corresponding problem of the plate has received but little attention. Mathieu discussed the problem as early as 1869 , but the method adopted is different from that followed in the present paper, the main object of which is to apply Whittaker's solutions $f$ of Mathieu's Equation to the problem of the elliptic plate. These solutions are really better suited for numerical calculations than the evaluation of infinite determinants.

\section{\$. The equation of motion and its solution.}

Let the thickness of the plate be $2 \alpha$, the volume density $\rho$ and Poisson's ratio $\sigma$. If $w$ be the transverse displacement, the equation of motion is +

$$
\frac{\partial^{2} w}{\partial t^{2}}+\frac{E \alpha^{2}}{3 \rho\left(1-\sigma^{2}\right)} \nabla^{4} w=0,
$$

where

$$
\nabla^{4} \equiv \frac{\partial^{4}}{\partial x^{4}}+2 \frac{\partial^{4}}{\partial x^{2} \partial y^{2}}+\frac{\partial^{4}}{\partial y^{4}}
$$

If $\frac{2 \pi}{p}$ be the period of oscillation, and $w$ varies as $\cos (p t+\epsilon)$, the equation (1) reduces to

$$
\nabla^{4} w-k^{4} w=0
$$

* Liouville, t. XIII, 1868.

+ Proc. Edin. Math. Soc., Vol. 32 (1913-14).

$\ddagger$ RaYleigii, Theory of Sound, Vol. I, Chap. X. 
where

$$
k^{4}=\frac{p^{2}}{c^{2}}, \quad c^{2}=\frac{E_{\left(\alpha^{2}\right.}}{3 \rho\left(1-\sigma^{2}\right)}
$$

From (2), we have $\left(\nabla^{2}+k^{2}\right)\left(\nabla^{2}-k^{2}\right) w=0$, and if $w_{1}$ and $w_{2}$ be respectively the solutions of

$$
\begin{aligned}
& \left(\nabla^{2}+k^{2}\right) w_{1}=0 \\
& \left(\nabla^{2}-k^{2}\right) w_{2}=0,
\end{aligned}
$$

then $w=A w_{1}+B w_{2}$ is a solution of $\left(\nabla^{+}-k^{4}\right) w=0$. Hence a solution of (1) is

$$
w=\left(A w_{1}+B w_{2}\right) \cos (p t+\epsilon)
$$

Putting $x+i y=h \cosh (\xi+i \eta)$, so that $\xi=$ const. give confocal ellipses with focal distance $2 h$, the equation (4) becomes

$$
\frac{\partial^{2} w_{1}}{\partial \xi^{2}}+\frac{\partial^{2} w_{1}}{\partial \eta^{2}}+h^{2} k^{2}\left(\cosh ^{2} \xi-\cos ^{2} \eta\right)=0
$$

Let $w_{1}=F(\xi) G(\eta)$, where $F(\xi)$ is a function of $\xi$ only and $G(\eta)$ a function of $\eta$ only. We get

where

$$
\left.\begin{array}{l}
\frac{\partial^{2} F}{\partial \xi^{2}}+(16 q \cosh 2 \xi-N) F=0 \\
\frac{\hat{\sigma}^{2} G}{\partial \eta^{2}}-(16 q \cos 2 \eta-N) G=0
\end{array}\right\}
$$

If we put $\xi=i\left(z+\frac{\pi}{2}\right), \eta=z+\frac{\pi}{2}$ respectively in the equations (8), they both reduce to the equation

$$
\frac{\partial^{2} u}{\partial z^{2}}+(a+16 q \cos 2 z) u=0
$$

The solution of this equation given by Whittaker is

$$
u=\Lambda(z)=e^{\mu z} u(\hat{\imath}),
$$

where $u(z)$. is a periodic function of $z$, and $\mu$ is given by the power series in $q$

$$
\mu=4 q \sin 2 \sigma-12 q^{3} \sin 2 \sigma-12 q^{4} \sin 4 \sigma+\ldots ;
$$


in this equation $\sigma$ is a parameter connected with the parameters $\alpha$ and $q$ of the differential equation by the relation

$a=1+8 q \cos 2 \sigma+(-16 q+8 \cos 4 \sigma) q^{2}-8 q^{3} \cos 2 \sigma+\ldots$

and $u(z)$ is given by the Fourier series

$$
\begin{aligned}
u(z)=\sin (z-\sigma) & +a_{3} \cos (3 z-\sigma)+b_{3} \sin (3 z-\sigma)+a_{3} \cos (5 z-\sigma) \\
& +b_{5} \sin (5 z-\sigma)+a_{7} \cos (7 z-\sigma)+\ldots, \ldots \ldots(14)
\end{aligned}
$$

where the coefficients are given by the power-series in $q$

$b_{3}=q+q^{2} \cos 2 \sigma+\left(-\frac{14}{3}+5 \cos 4 \sigma\right) q^{3}+\left(-\frac{74}{4} \cos 2 \sigma+7 \cos 6 \sigma\right) q^{4}+\ldots$

$a_{3}=3 q^{2} \sin 2 \sigma+3 q^{3} \sin 4 \sigma+\left(-\frac{2 \pi g}{9} \sin 2 \sigma+9 \sin 6 \sigma\right) q^{4}+\ldots$

$b_{5}=\frac{1}{3} q^{2}+\frac{4}{9} q^{3} \cos 2 \sigma+\left(-\frac{155}{54}+\frac{4}{9} \frac{2}{7} \cos 4 \sigma\right) q^{4}+\ldots$

$a_{5}=\frac{14}{8} q^{3} \sin 2 \sigma+\frac{44}{2} q^{4} \sin 4 \sigma+\ldots$

$b_{i}=\frac{1}{18} q^{3}+\frac{1}{12} q^{4} \cos 2 \sigma+\ldots$, etc.,

A second solution is obtained by putting $-\sigma$ for $\sigma$. Hence the complete solution is

$$
u=A \Lambda(z, \sigma, q)+B \Lambda(z,-\sigma, q)
$$

The solution is purely periodic when $\mu=0$. Let $\sigma=s$ be any one of the values of $\sigma$ which makes $\mu=0$. Then the solution (16) reduces to a single term and the general solution must be taken as

$$
u=A \Lambda(z, s, q)+B \Lambda_{1}(z, s, q),
$$

where $\Lambda_{1}(z, s, q)$ is the elliptic cylinder function of the second kind.*

In our problem, $G(\eta)$ must be a purely periodic function; hence we have

$$
G(\eta)=\Lambda\left(\eta-\frac{\pi}{2}, s, q\right),
$$

since the elliptic cylinder function of the second kind is not periodic Also

$$
\begin{aligned}
F(\xi) & =A \Lambda\left[-i \xi-\frac{\pi}{2}, s, q\right]+B \Lambda_{1}\left[-i \xi-\frac{\pi}{2}, s, q\right] \\
& =A H_{1}(\xi)+B F_{2}(\xi),
\end{aligned}
$$

* E. L. INce. Proc. Eudin. Math. Soc., Vol. 33, p. 2. 
where for brevity, we have put

$$
\begin{aligned}
& \Lambda\left[-i \xi-\frac{\pi}{2}, s, q\right]=H_{1}(\xi) \\
& \Lambda_{1}\left[-i \xi-\frac{\pi}{2}, s, q\right]=F_{2}(\xi) .
\end{aligned}
$$

Hence

$$
w_{1}=G(\eta)\left\{A F_{1}(\xi)+B F_{2}(\xi)\right\}
$$

To obtain the solution of $(5)$, we take $w_{2}=P(\xi) Q(\eta)$, where $P(\xi)$ is a function of $\xi$ only and $Q(\eta)$ is a function of $\eta$ only, and setting $a=N+\frac{1}{2} h^{2} k^{2}, q=\frac{1}{32} h^{2} k^{2}$ instead of (9), we get

$$
\begin{aligned}
& P(\xi)=C \Lambda(i \xi, s, q)+D \Lambda_{1}(i \xi, s, q), \\
& Q(\eta)=\Lambda(\eta, s, q),
\end{aligned}
$$

since $Q(\eta)$ must be periodic.

Putting, for brevity,

$$
\begin{aligned}
& \Lambda(i \xi, s, q)=P_{1}(\xi) \\
& \Lambda_{1}(i \xi, s, q)=P_{2}(\xi),
\end{aligned}
$$

we have

$$
w_{2}=Q(\eta)\left\{C P_{1}(\xi)+D P_{2}(\xi)\right\}
$$

Hence we obtain finally

$$
\begin{aligned}
w=\left[G ( \eta ) \left\{A F_{1}^{\prime}(\xi)\right.\right. & \left.+B F_{2}(\xi)\right\} \\
& \left.+Q(\eta)\left\{C P_{1}(\xi)+D P_{2}(\xi)\right\}\right] \cos (p t+\epsilon)
\end{aligned}
$$

\$3. The case of the complete elliptic disc.

Let $\xi=\xi_{0}$ be the boundary of the ellipse, so that we have for a rigidly clamped boundary $\xi=\xi_{0}, w=\frac{\partial w}{\partial \dot{\xi}}=0$. Hence from (20) we have

$G(\eta)\left\{A F_{1}\left(\xi_{0}\right)+B F_{2}\left(\xi_{0}\right)\right\}+Q(\eta)\left\{C P_{1}\left(\xi_{0}\right)+D P_{2}\left(\xi_{0}\right)\right\}=0$

$G(\eta)\left\{A F_{1}^{\prime}\left(\xi_{0}\right)+B F_{2}^{\prime}\left(\xi_{11}\right)\right\}+Q(\eta)\left\{C P_{1}^{\prime}\left(\xi_{0}\right)+D P_{2}^{\prime}\left(\xi_{0}\right)_{j}=0\right\}$

where $F_{1}^{\prime}\left(\xi_{0}\right), \ldots$ are the values of $\frac{\partial F_{1}(\xi)}{\partial \xi}, \ldots$ when $\xi=\xi_{0}$.

Since the equations (21) must be true for all values of $\eta$, the coefficients of $G(\eta)$ and $Q(\eta)$ must separately vanish. We there- 
fore obtain, after the elimination of $A, B, C, D$, the two equations

$$
\left|\begin{array}{ll}
F_{1}\left(\xi_{0}\right) & F_{2}\left(\xi_{0}\right) \\
F_{1}^{\prime}\left(\xi_{0}\right) & F_{2}^{\prime}\left(\xi_{0}\right)
\end{array}\right|=0,\left|\begin{array}{ll}
P_{1}\left(\xi_{0}\right) & P_{2}\left(\xi_{0}\right) \\
P_{1}^{\prime}\left(\xi_{0}\right) & P_{2}^{\prime}\left(\xi_{0}\right)
\end{array}\right|=0
$$

These equations give two types of vibration.

\section{\$4. The case of the elliptic annulus.}

If the boundaries are the confocal ellipses $\xi=\xi_{0}$ and $\xi=\xi_{1}$, we must have $w=\frac{\partial w}{\partial \hat{\xi}}=0$ for these values of $\dot{\xi}$. Hence, we get, in addition to (22), the following relations :

$$
\begin{aligned}
& G(\eta)\left\{A F_{1}\left(\xi_{1}\right)+B F_{2}^{\prime}\left(\xi_{1}\right)\right\}+Q(\eta)\left\{C P_{1}\left(\xi_{1}\right)+D P_{2}\left(\xi_{1}\right)\right\}=0 \\
& G(\eta)\left\{A F_{1}^{\prime}\left(\xi_{1}\right)+B F_{2}^{\prime}\left(\xi_{1}\right)\right\}+Q(\eta)\left\{C P_{1}^{\prime}\left(\xi_{1}\right)+D P_{2}^{\prime}\left(\xi_{1}\right)\right\}=0 .
\end{aligned}
$$

These four equations must hold for all values of $\eta$. Hence the eight coefficients of $G(\eta)$ and $Q(\eta)$ should all vanish. Thus, on eliminating $A, B, C, D$, we get

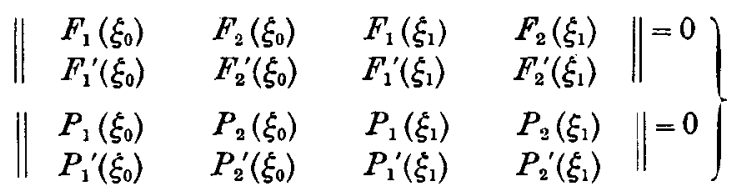

These give us the frequency equations. It appears from the form of these equations that they are fairly simple and numerical calculations can be effected by means of (12), (13), (14) and (15).

\section{\$5. An important particular care.}

There is an infinite number of values of $s$ (i.e. the values of $\sigma$ ) which make $\mu=0$. And corresponding to every value of $s$, we shall obtain a set of frequency-equations of the type (22) or (23).

$$
\text { Since } q= \pm \frac{h^{2}}{32} \sqrt{\frac{3 \rho\left(1-\sigma^{2}\right)}{E \alpha^{2}}} \cdot p,
$$

where $\sigma$ is the Poisson's ratio, it is evident that $q$ is a small quantity for all ordinary substances, and hence the powers of $q$ beyond the second may be omitted in obtaining an approximate 
solution. In this case we have $\mu=q \sin 2 \sigma$ and for $\mu=0$, we have $s=0$ or $\frac{\pi}{2}$. In either case, the solutions $\Lambda(z, s, q)$ and $\Lambda_{1}(z, s, q)$ assume simple forms.

In fact, we get, up to the second power of $q$ $\Lambda(z, o, q)=\sin z+\left(q+q^{2}\right) \sin ? z+\frac{1}{2} q^{2} \sin 5 z$ $\Lambda_{1}(z, o, q)=-8 q z \Lambda(z, o, q)+\cos z+q \cos 3 z+q^{2}\left(\frac{1}{3} \cos 5 z-5 \cos 3 z\right)$ $\Lambda(i z, o q)=-i\left[\sinh z+\left(q+q^{2}\right) \sinh 3 z+\frac{1}{2} q^{2} \sinh 5 z\right]$ $\Lambda_{1}(i z, o, q)=-8 q . i z . \Lambda(i z, o, q)+\cosh z+q \cosh 3 z$ $+q^{2}\left(\frac{1}{3} \cosh 5 z-5 \cosh 3 z\right)$.

As a first approximation, we may take

$$
\begin{aligned}
& \Lambda(z, o, q)=\sin z+q \sin 3 z \\
& \Lambda_{1}(z, o, q)=-8 q z \sin z+\cos z+q \cos 3 z \\
& \Lambda(i z, o, q)=-i(\sin z+q \sinh 3 z) \\
& \Lambda_{1}(i z, o, q)=-8 q z \sinh z+\cosh z+q \cosh 3 z .
\end{aligned}
$$

\section{\$6. The frequency equation.}

The simplest type for the complete elliptic disc $\xi=\xi_{0}$ is giren by the equation

$$
P_{1}\left(\xi_{0}\right) P_{2}^{\prime}\left(\xi_{0}\right)-P_{1}^{\prime}\left(\xi_{0}\right) P_{2}\left(\xi_{0}\right)=0 .
$$

Substituting for $P_{1}\left(\xi_{0}\right), P_{1}^{\prime}\left(\xi_{0}\right), P_{2}\left(\xi_{0}\right), P_{2}^{\prime}\left(\xi_{0}\right)$ from above, we get, retaining the first powers of $q$ only,

$$
q=\frac{1}{4\left(1-2 \cosh 2 \xi_{0}\right)}
$$

Hence, if $n$ be the frequency we get from (24) and (25)

$$
n=\frac{4 \alpha}{\pi \iota^{2}} \sqrt{\frac{E}{3 \rho\left(1-\sigma^{2}\right)}} \frac{1}{\left(4 \cosh ^{2} \xi_{0}-3\right)}
$$

\$7. Numerical Results.

A few numerical results are added below. In the different cases considered, the material and the eccentricity of the ellipse have been varied, but the thickness and the focal distance are 
unaltered. The frequency is obtained as the number of vibrations per second. The units employed are the usual c. g. s. units.

$\begin{array}{cccccccc}\text { Material } & \begin{array}{c}\rho \\ \text { Density }\end{array} & \begin{array}{c}E \\ \text { Young's } \\ \text { Modulus }\end{array} & \begin{array}{c}\sigma \\ \text { Poisson's } \\ \text { ratio }\end{array} & \begin{array}{c}2 a \\ \text { Thick- }\end{array} & \begin{array}{c}2 h \\ \text { ness }\end{array} & \begin{array}{c}e \\ \text { Focal }\end{array} & \begin{array}{c}n \\ \text { Eccen- }\end{array} \\ \text { Steel Fre- } \\ \text { tricity }\end{array}$

